\title{
Optimale Methoden zur Interpolation von Umweltvariablen in Geographischen Informationssystemen
}

\begin{abstract}
Summary
In Geographic Information Systems there are many applications where the objects of study can only be described by sampling information at selected point locations and by subsequent interpolation. Quite often inappropriate methods are used for the interpolation of sparse environmental data which do not take into account the characteristics of the data themselves. This paper presents the case for more widely using Kriging-methods in environmental data approximation. It gives a short theoretical overview of Kriging and the interpretation of variograms and goes on with a discussion of more advanced concepts (Co-Kriging, disjunctive Kriging), some examples and the illustration of the use of Kriging within a GIS environment.
\end{abstract}

\section{Einleitung}

Viele Geographische Informationssysteme arbeiten ausschließlich mit exakt definierten Objekten, die durch Linien oder Polygone dargestellt werden können (z.B. in Katastersystemen oder der thematischen Kartographie), aber es gibt Anwendungen, deren Studienobjekte mit Hilfe von regulär oder irregulär angeordneten Stichprobenpunkten erfaßt und deren geographische Form lediglich durch Approximation bestimmt werden kann. Im Bereich der natürlichen Ressourcen, der Landbewertung und von Schadstoffuntersuchungen können die Eigenschaften von Boden, Grundwasser oder Atmosphäre oft nur durch Erhebungen an selektiven Punkten erfaßt werden. Um diese Daten zu verbinden, z.B. zu Flächen einer Landnutzungskarte, ist es notwendig, eine Oberfläche durch die Datenpunkte zu interpolieren und diese entweder durch Isolinien oder Werte eines feinen Rasters darzustellen. Die resultierende Oberfläche kann dann zur Darstellung verwendet oder zur weiteren Analyse anderen Informationsebenen überlagert werden. Die Figur 1 zeigt den Informationsfluß von den Datenpunkten bis zur Eingabe von Informationsebenen in GIS.

Das Problem der möglichst genauen Interpolation zwischen Beobachtungspunkten stellt sich auch in der Vermessung, wo die Erdoberfläche modelliert wird, um digitale Geländemodelle (DGM) aufzubauen. Folgendes sind die Hauptunterschiede zwischen Hypsometrie und der Interpolation von Boden-, Geologie-, Wasser- oder Luftqualitätsdaten: Im ersten Bereich können die Oberflächenformen beobachtet und überall visuell überprüft werden, während die Oberflächen chemischer oder phy-
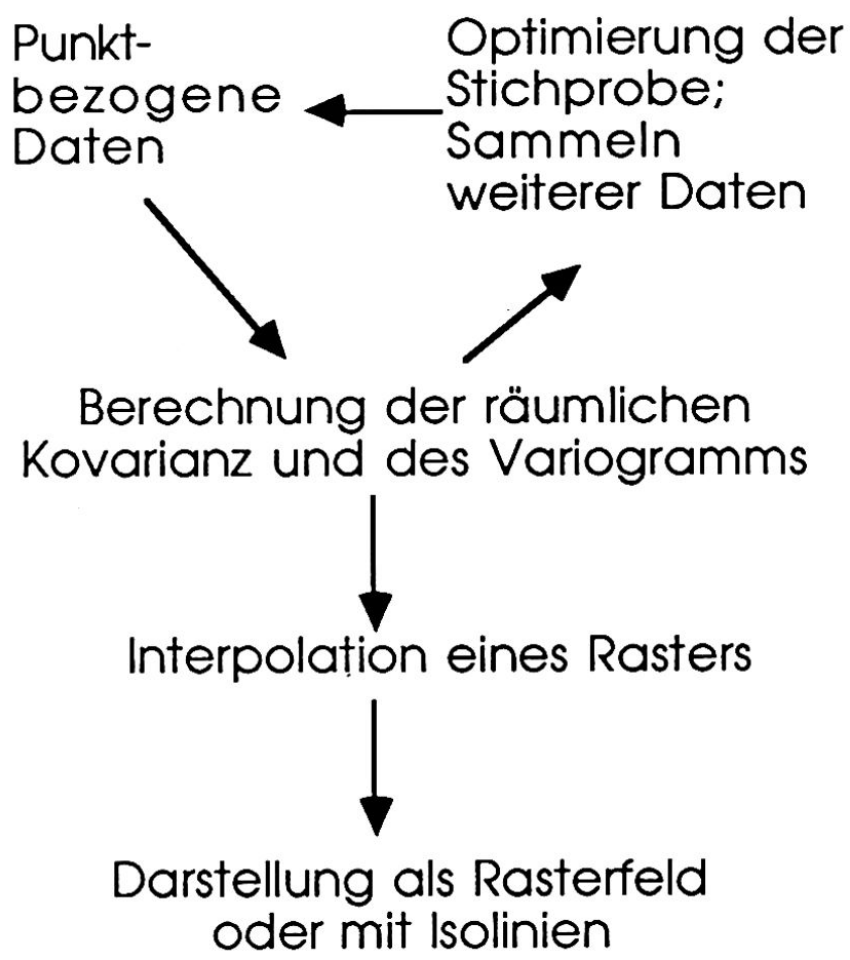

\section{Eingabe in ein GIS}

Figur 1

sikalischer Eigenschaften der Umwelt nie direkt sichtbar sind. Zweitens sind die Kosten für die Erhebung und Auswertung (evtl. Laborauswertungen) einzelner Beobachtungswerte bei Umweltdaten in der Regel wesentlich höher als die photogrammetrische Erhebung mit Stereopaaren. Drittens weisen die durch Stichprobenerfassung ermittelten Daten in der Regel bedeutend gröBere Fehler auf als die Höhendaten. Die Größe dieser Fehler hängt einerseits zusammen mit der Tatsache, daß lediglich Stichproben oft geringer Größe verwendet werden, andererseits mit Unsicherheiten der Labormethoden sowie unbekannten, kleinräumigen Variationen

P.A. Burrough, Prof., Dr., Institut für Physische Geographie, Fakultät der Geographischen Wissenschaften, Universität Utrecht, Postfach 80.115, 3508 TC Utrecht/NL 
zwischen den Stichprobenpunkten. So müssen die Interpolationsmethoden für Umweltstudien an geringe $\mathrm{Da}$ tenmengen und fehleranfällige Situationen angepaßt sein, wo die Resultate lediglich durch stichprobenartige Kontrollen überprüft werden können. Unter diesen Bedingungen sollen Interpolationsverfahren gewählt werden, die aus den wenigen und teuren Datenpunkten eine möglichst gute Oberfläche modellieren.

Es existieren verschiedene routinemäßig eingesetzte Interpolationsmethoden. Beispiele sind globale und lokale polynomiale Trendmethoden, exakte oder glättende Spline-Berechnungen, die Benutzung von ThießenPolygonen oder die Interpolation mit inverser Distanzgewichtung (vgl. BURROUGH 1986, DAVIS 1986), wovon einige häufig in kommerziellen GIS integriert sind. Alle diese Techniken berechnen Werte im Zwischenbereich von Stichprobenpunkten in der einen oder anderen Form als globale oder lokale gewichtete Mittel aus den Datenpunkten und einem Distanzmaß zu diesen. Es handelt sich dabei durchwegs um Methoden, die durch die Charakteristik der Daten nicht beeinflußt werden (i.e. es sind sog. externe Methoden); folglich sind ihre Resultate selten optimal. So können exakte Methoden wie lineare Interpolation in triangulierten Netzen (TIN) fehlerhafte und verzerrte Resultate erzeugen, und das Einpassen von exakten Spline-Oberflächen auf Facetten mag zu überlappenden Isolinien oder übersteilen Verknüpfungen zwischen den Facetten führen (vgl. BURROUGH 1986, Fig. 3.1).

Die Problematik der Verwendung externer Methoden liegt daran, daß Interpolationsparameter geschätzt werden müssen, ohne dabei die Daten zu berücksichtigen. So können bei der inversen Distanz-Methode der Exponent der Gewichtungsfunktion und die Zahl der verwendeten Datenpunkte frei gewählt werden. Folglich wird das Resultat der Interpolation weitgehend von diesen Parameterwerten bestimmt, ohne dabei die räumliche Variation optimal wiederzugeben. Diese Methoden erzeugen immer ein Resultat, auch wenn die erfaßte Oberfläche so stark variiert, daß sie besser als Rauschen dargestellt wird. Dazu kommt, daß diese Allerweltstechniken selten die Anisotropie der Datenoberfläche mit berücksichtigen, wie sie oft in richtungsabhängiger Variation von Mustern vorliegt. Es ist offensichtlich, daß bei gleichbleibender Datenpunktmenge besser Interpolationsresultate erzielt werden können, wenn die Interpolationsmethoden und die Schätzparameter zuerst auf die Variation der zu bearbeitenden Daten abgestimmt werden.

Um diesem Problem zu begegnen, haben der französische Geomathematiker G. Matheron und der südafrikanische Mineningenieur D.G. Krige die Theorie der «Regionalisierten Variablen» und das zugehörige Interpolationsverfahren mit dem Namen Kriging entwickelt. Diese Methoden fanden bisher Anwendung in Minenindustrie, Grundwasserkartierung, Bodenkartierung und verwandten Gebieten (AKIN UND SIEMES 1988, MATHERON 1971, JOURNEL UND HUIJGBREGTS 1978, WEBSTER
UND OLIVER 1990), doch sie finden nun auch Eingang in Geographische Informationssysteme.

\section{Kurze Einführung in die Theorie der Regionalisierten Variablen und das Kriging}

Bei unserer Darstellung der Theorie der Regionalisierten Variablen soll nur ein Minimum an mathematischen Formulierungen verwendet werden. Eine exaktere Darstellung findet der Leser in den oben erwähnten Texten. Die Theorie der Regionalisierten Variablen geht davon aus, daß die räumliche Variation einer Variablen in drei Komponenten aufgegliedert werden kann:

a) eine strukturelle Komponente (konstantes Mittel oder konstanter Trend),

b) eine zufällige, aber räumlich korrelierte Komponente, und

c) einen Residualfehler oder das zufällige Rauschen.

Für ein $\mathrm{x}$, das eine Position in 1, 2, oder 3 Dimensionen darstellt, gilt also für den Wert einer Variablen $\mathrm{Z}$

$Z(x)=m(x)+\varepsilon^{\prime}(x)+\varepsilon^{\prime \prime}$

wobei $\mathrm{m}(\mathrm{x})$ der strukturellen Komponente, $\varepsilon^{\prime}(\mathrm{x})$ dem stochastischen, lokal variierenden, räumlich abhängigen Residual von $\mathrm{m}(\mathrm{x})$ und $\varepsilon$ " dem räumlich unabhängigen Rauschen mit einem Mittel von Null und einer Varianz von $\sigma^{2}$ entspricht.

Die strukturelle Komponente $\mathrm{m}(\mathrm{x})$ kann entweder durch den Mittelwert über das gesamte erfaßte Gebiet oder, wenn ein genereller Trend vorliegt, durch eine Trendoberfläche niedriger Ordnung repräsentiert werden. Um mögliche Fehler zu vermeiden, muß die zu verwendende Trendoberfläche sorgfältig ausgewählt werden. Für die weiteren Betrachtungen subtrahieren wir die strukturelle Komponente (Mittelwert oder Trend) von den Ausgangsdaten und berücksichtigen nur die resultierenden Residualwerte.

Nach der Theorie der Regionalisierten Variablen nimmt man an, daß die Varianz der Differenzen der Attributwerte zwischen Beobachtungspunkten nur von deren $\mathrm{Di}$ stanz abhängt. Dies wird formuliert durch die sog. Intrinsische Hypothese, die als Bedingungen die Stationarität der Differenz und der Varianz der Differenzen annimmt. Sind die strukturellen Effekte eliminiert, so ist die Restvarianz homogen, d. h., Varianzen zwischen unterschiedlichen Standorten sind lediglich eine Funktion der Distanz zwischen ihnen. Als Maß für diese Varianz in Abhängigkeit der Distanz wird die sog. Semivarianz $\gamma$ verwendet, die sich anhand der Höhenunterschiede sämtlicher paarweise verglichener Beobachtungspunkte bestimmen läßt und in einem Variogramm dargestellt werden kann (Figur 2). Im Variogramm wird die Semivarianz $\gamma$ als Funktion der Distanz zwischen benachbarten Standorten ausgedrückt, und die darin enthaltene Information wird nun dazu verwendet, optimale Gewichte für die Interpolation zu bestimmen. 


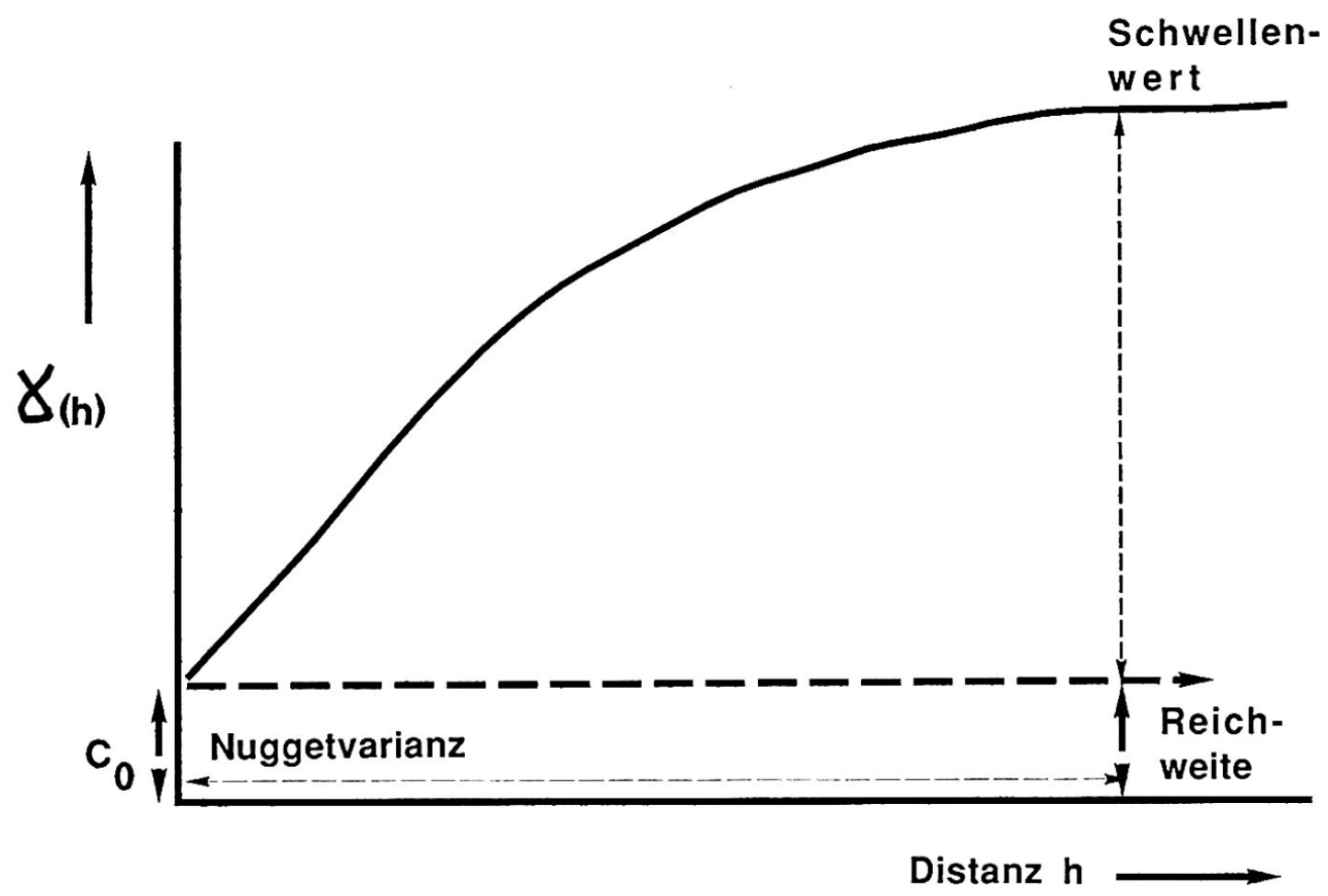

Figur 2

Die Figur 2 stellt ein typisches experimentelles Variogramm für Bodendaten dar. Die empirisch festgestellten Semivarianzwerte für alle Paare von Beobachtungspunkten sind in Abhängigkeit von ihrer Distanz vorerst ins Diagramm eingetragen und dann durch eine passende Kurve ersetzt worden. In dieser Kurve sind folgende wichtige Elemente festzuhalten. Erstens flacht sich die Kurve bei einem großen Wert der Distanz h ab. Dieser horizontale Teil der Kurve wird als Schwellenwert (engl. sill)bezeichnet, die Stelle auf der X-Achse, an der die Kurve abflacht, als Reichweite (engl. range).Zweitens steigt innerhalb der Reichweite die Kurve kontinuierlich an und beschreibt so die Abhängigkeit der Semivarianz $\gamma$ von der Distanz h. Je näher zwei Raumpunkte beieinanderliegen, um so ähnlicher sind sie sich; das bedeutet, daß nahe gelegene Datenpunkte für die Interpolation ein gröBeres Gewicht erhalten sollen. Jenseits der Reichweite wird aber die Schätzung der Varianz der Differenzen unabhängig von der Distanz oder - in anderen Worten ausgedrückt - können Datenpunkte, die von einem zu interpolierenden Punkt weiter entfernt sind als die Reichweite, keinen sinnvollen Beitrag für die Schätzung seines Wertes leisten. Die Reichweite gibt also Auskunft über die Größe des Suchfeldes für Datenpunkte, die für die Interpolation verwendet werden sollen. Als drittes Merkmal zeigt die Figur 2, daß die modellierte Kurve nicht durch den Systemursprung geht, sondern die Y-Achse an einem positiven Wert $\mathrm{C}_{0}$, der sog. Nuggetvarianz (engl. nugget), trifft. An sich sollte die Semivarianz $\gamma$ für $\mathrm{h}=0$ verschwinden, doch die empirisch bestimmte Kurve weicht von diesem idealen Verhalten wegen der zufälli- gen Fehler oder des Rauschens ab. Die Nuggetvarianz gibt uns also eine Schätzung für die zufällig verteilte Restvarianz $\varepsilon$ ". Es handelt sich um die Varianz, die auf Meßfehler und kleinräumige Variationen zurückzuführen ist, die aufgrund der Abstände der Stichprobenpunkte nicht erklärt werden können.

Die Form des Variogramms ist sehr bedeutsam; sie hängt auch ab von der Methode, mit der es berechnet wird. In der Praxis werden oft sphärische oder exponentielle Modelle, aber auch lineare, zirkuläre oder Gaußsche Modelle verwendet (Details s. JOURNEL und HUIJBREGTS 1978). Die Form des Variogramms modelliert die räumliche Abhängigkeit zwischen den Datenpunkten als Funktion ihrer Distanz. Diese Funktion ist ein Analogon zur inversen Distanzfunktion, aber sie ist aus den erfaßten Datenpunkten abgeleitet worden. Die Variogrammfunktion liefert deshalb die beste lokale Schätzung für die Gewichtung bei der Vorhersage der lokalen Mittelwerte.

Kriging ist eine exakte Interpolationsmethode, d.h., sie kann für die Schätzung von Einzelpunkten eingesetzt werden (Punkt-Kriging). Oft ist es aber sinnvoller, lokale Mittel für ein Gebiet, d. h. mittlere Werte für Flächeneinheiten oder Rasterzellen zu schätzen (Block-Kriging), die dann zur kartographischen Modellierung mit anderen Dateien kombiniert werden können. Für Block-Kriging gilt für die Vorhersage des Wertes $\hat{Z}\left(X_{\mathrm{B}}\right)$ für ein $\mathrm{Ge}$ biet B (Figur 3):

$$
\begin{gathered}
\hat{Z}\left(X_{B}=\sum n \lambda_{i} Z\left(X_{i}\right)\right. \\
i=1
\end{gathered}
$$




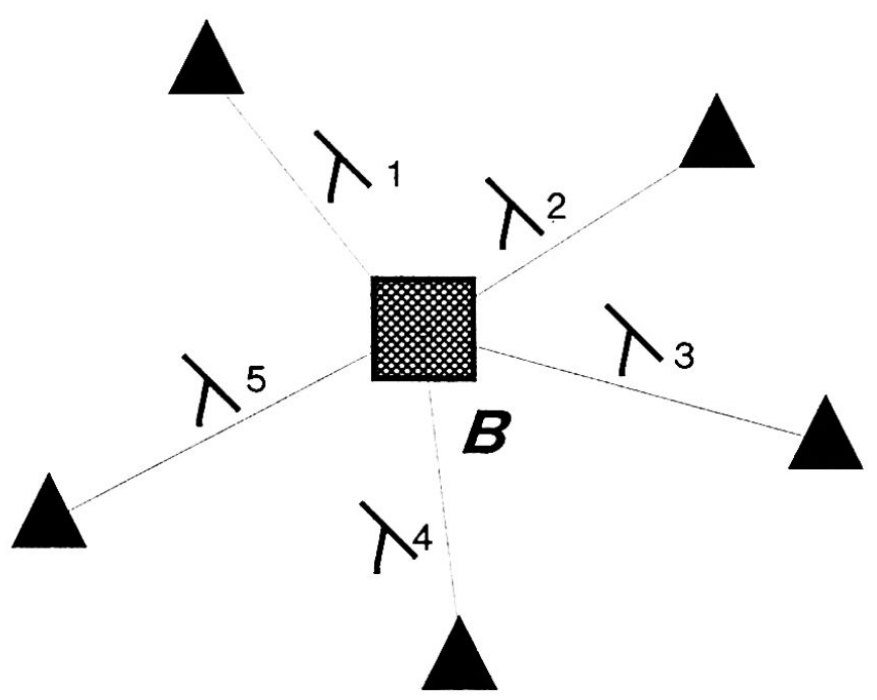

Figur 3

wobei die Summe der Gewichte $\lambda_{i}$ eins ist und die Gewichte $\lambda_{\mathrm{i}}$ so gewählt werden, daß die Schätzung von $\hat{Z}\left(X_{B}\right)$ unverfälscht und die Varianz $\sigma_{B}{ }^{2}$ des Blockmittels minimal ist (d.h. kleiner als jede andere Linearkombination der beobachteten Werte).

Für die Berechnung der Gewichte $\lambda_{i}$ werden die Semivarianzen $\gamma$ des Variogramms verwendet, wobei Werte für sämtliche Kombinationen der berücksichtigten Datenpunkte $\gamma\left(X_{\mathrm{i}}, X_{\mathrm{i}}\right)$, der Distanzen zwischen Datenpunkten und Blockmitte $\gamma\left(X_{i}, X_{B}\right)$ wie auch die mittlere Varianz $\gamma\left(X_{B}, X_{B}\right)$ innerhalb des Blocks $B$ berücksichtigt werden. Die geschätzten Varianzen sind beim Block-Kriging in der Regel bedeutend kleiner als beim Punkt-Kriging, da die blockinterne Varianz subtrahiert werden kann.

Falls die Nuggetvarianz $\varepsilon$ "die lokale Variation des experimentellen Variogramms so dominiert, daß die Kurve im Bereich der Reichweite nicht signifikant ansteigt, so sind die Daten so schlecht, daß eine Interpolation nicht sinnvoll ist. In dieser Situation stellt die Horizontalebene auf der Höhe des Mittels aller Datenpunkte die beste Schätzung für die Oberfläche dar. Falls das Variogramm für alle Richtungen im Raum Gültigkeit besitzt, wird es als isotropisch bezeichnet. Vermutet man richtungsabhängig unterschiedliches Verhalten der Daten, kann für verschiedene Richtungen je ein Variogramm berechnet werden (z. B. für N-S- und E-W-Richtungen). Diese Variogramme geben Auskunft über die Form und Orientierung der Fenster, anhand deren die Punkte für die Interpolation ausgewählt werden sollen.

\section{Anwendungen und weiterführende Konzepte}

In kartographischen Anwendungen sollen oft, ausgehend von einer Anzahl von Stichprobenpunkten (frei oder in einem Raster angeordnet), Werte für die Zwi- schenbereiche geschätzt werden, die dann als (feiner) Raster oder mit Hilfe von Isolinien dargestellt werden. Neben der Kartierung der interpolierten Oberfläche selbst ermöglicht es aber das Kriging auch, die Schätzungswerte der Varianz $\sigma^{2}$ kartographisch darzustellen, also Aussagen über die Zuverlässigkeit der Interpolation zu machen. Figur 4 zeigt das Resultat einer Interpolation mit Block-Kriging für den prozentualen Sandanteil einer Farm von $750 \mathrm{~m} \times 750 \mathrm{~m}$ in Alberta, Canada (MAC MILLAN 1987). Die Originaldaten wurden in einem 60-m-Raster erhoben (dargestellt durch Sternsymbole); daraus wurde ein 30-m-Raster interpoliert und mit einem Isolinienprogramm dargestellt (Figur 4a). Figur $4 b$ zeigt die Schätzung der Interpolationsfehler; diese sind in der Nähe der Datenpunkte minimal und wachsen stark an in den Gebieten, wo Datenpunkte fehlen (NE- und NW-Ecken). In Geographischen Informationssystemen sind in der Regel mehrere Datenebenen mit unterschiedlichen Datencharakteristiken abgespeichert. So kann eine Informationsebene $\mathrm{U}$ mit einer geringen Zahl von Datenpunkten erfaßt worden sein, da die Datenerfassung aufwendig und teuer ist, während für eine andere Ebene V ein engeres Maschennetz von Erfassungspunkten vorliegt. Falls $\mathrm{U}$ und $\mathrm{V}$ miteinander korreliert sind, kann die Information aus $\mathrm{V}$ dazu verwendet werden, die Interpolation U zu verbessern. Dieses Verfahren wird als Co-Kriging bezeichnet. Es verwendet Informationen aus den Variogrammen der beiden Ebenen $\mathrm{U}$ und $\mathrm{V}$ und modelliert in einem Co-Variogramm die gemeinsame Co-Variation beider Variablen (MCBRATNEY und WEBSTER 1983). LEENAERS ET AL. (1989) kartierten mit Hilfe von Co-Kriging die Schwermetallanteile (Cadmium, Blei und Zink) des Oberbodens des Einzugsgebietes der Geul in Süden der Niederlande. Die Schwermetalle wurden durch Schwemmprozesse aus Minen der Einzugsgebiete in die Schwemmebene transportiert. Da diese Verunreinigungen an feine Sedimentpartikel angelagert sind, werden sie nur sehr langsam aus der Suspension entlassen; folglich besteht eine starke negative Korrelation zwischen der relativen Höhe der Flußebenen-Oberfläche und der Schwermetallkonzentration. Die Resultate der aufwendigen Laboruntersuchungen der Schwermetallkonzentrationen konnten aufgewertet werden durch Co-Kriging mit Hilfe von Höheninformationen aus der topographischen Karte 1:10000. Schwermetalldaten des Oberbodens wurden an 154 Stellen erhoben und mit Hilfe von insgesamt 463 Oberflächenpunkten verarbeitet. Die Figur 5 zeigt, daß mit Hilfe von Co-Kriging eine glattere Fehleroberfläche der Zinkkonzentration mit geringeren Schätzfehlern resultiert (5b) als mit Kriging der Schwermetallwerte allein $(5 a)$.

Methoden der optimalen Interpolation können auch verwendet werden, um zu bestimmen, ob der Wert eines Attributes einen bestimmten Grenzwert übertrifft oder nicht. Mit Hilfe des sog. disjunktiven Kriging können einerseits binäre Karten (mit je einer Signatur für Gebiete oberhalb und unterhalb des Grenzwertes) und anderseits Wahrscheinlichkeitsmatrizen für das Überschreiten 
des Grenzwertes berechnet werden (WEBSTER und OLIVER 1989).

Da mit Hilfe von Kriging die Aussagen über die räumliche Verteilung der Fehlergröße gemacht werden können, kann man diese Methode auch zur Optimierung der Stichprobenerhebung einsetzen. Ist das Variogramm bekannt, können daraus Strategien für Auswahl der Datenpunktkonfiguration zur Minimierung der Interpolationsfehler abgeleitet werden. Für mehr Details siehe MCBRATNEY ET AL. (1981).

Kriging-Verfahren finden schließlich bei der Analyse natürlicher Ressourcen innerhalb von Geographischen Informationssystemen eine wichtige Anwendung. In der Regel werden mehrere Informationsebenen $(X, Y, \ldots)$ oft in Rasterstruktur dargestellt - in einem GIS mit mathematischen Modellen analysiert, indem aus Zellenwerten der einzelnen Informationsebenen mit Hilfe von logischen, arithmetischen oder anderen Operatoren eine neue Variable U berechnet wird. Beispiele dazu sind die Berechnung des Erosionspotentials oder von Anbauerträgen aus Standortcharakteristiken eines Gebietes (vgl. BURROUGH 1989, DUMANSKI Und ONOREI 1989). Oft sind die Werte der Ursprungsdatenebenen nicht genau bekannt, sondern Schätzungen, denen ein Fehlerterm beigegeben ist $(X \pm \varepsilon x, Y \pm \varepsilon y, \ldots)$. Für quantitative Variablenebenen kann nun das Kriging die gewünschten Fehlerterme liefern. Für die Modellierung der Zielgröße $\mathrm{U}$ ist es wichtig, ebenfalls Anhaltspunkte über ihre Feh- lergröße $(U \pm \varepsilon u)$ zu erhalten. Dazu muß man neben der Fehlergröße der Originalebenen auch die Regeln der Fehlerfortpflanzung der entsprechenden Verknüpfungsoperationen kennen (BURROUGH 1986, HEUVELINK et al. 1989). Mit ihrer Hilfe kann nun nicht nur die Zuverlässigkeit der resultierenden Variablen $U$ insgesamt abgeschätzt, sondern auch entschieden werden, wie diese allenfalls verbessert werden kann. Sind die Fehler der Modellparameter zu groß, so sollten diese mit mehr Daten besser kalibriert werden. Liefert andererseits die Oberfläche einer bestimmten Informationsebene einen zu großen Beitrag zum Gesamtfehler, so sollte diese durch zusätzliches Datenmaterial verdichtet werden. Zur Verbesserung der Stichprobenstrategie kann wiederum das entsprechende Variogramm wichtige Hinweise liefern.

\section{Diskussion}

Kriging ist eine anspruchsvolle Technik, die ein beträchtliches Verständnis der Geostatistik voraussetzt; deren Anwendung lohnt sich aber sehr häufig, da sie in der Regel konsistentere Resultate mit geringeren Schätzfehlern bringt als Standard-Interpolationstechniken. Die Methode basiert auf einer Analyse der vorliegenden Daten, wobei die wichtigsten Charakteristiken der Daten im Variogramm zum Ausdruck kommen. Folgende Informationen sind darin enthalten:

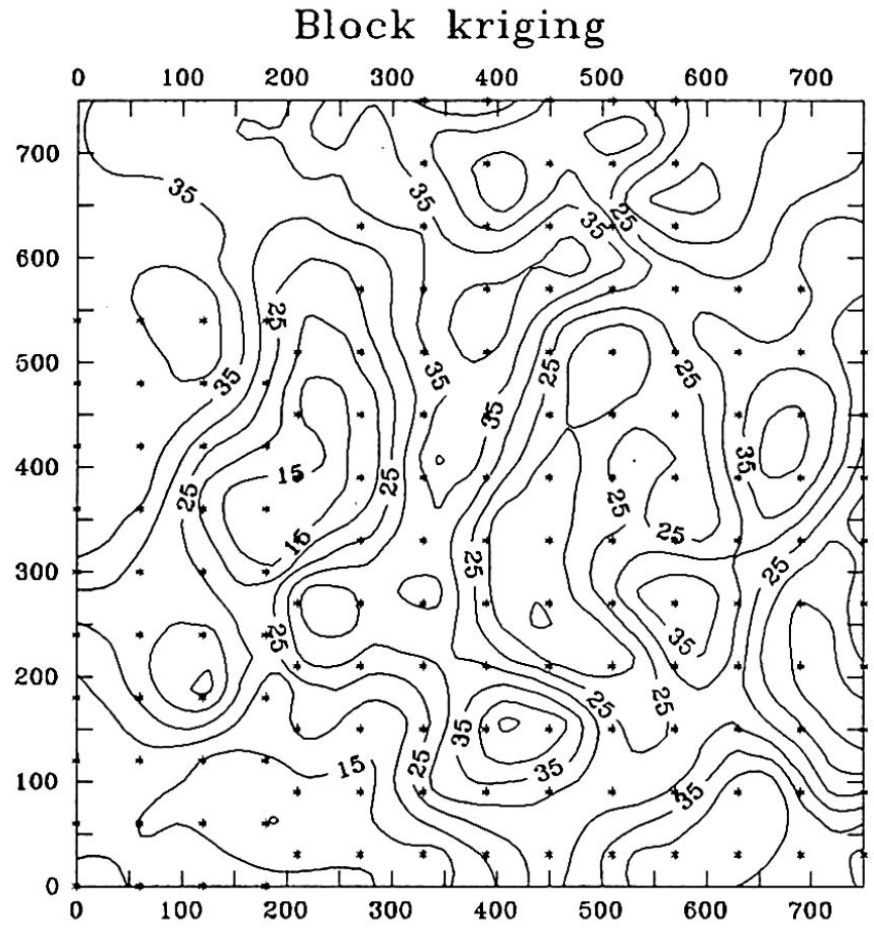

Figur $4 \mathrm{a}$

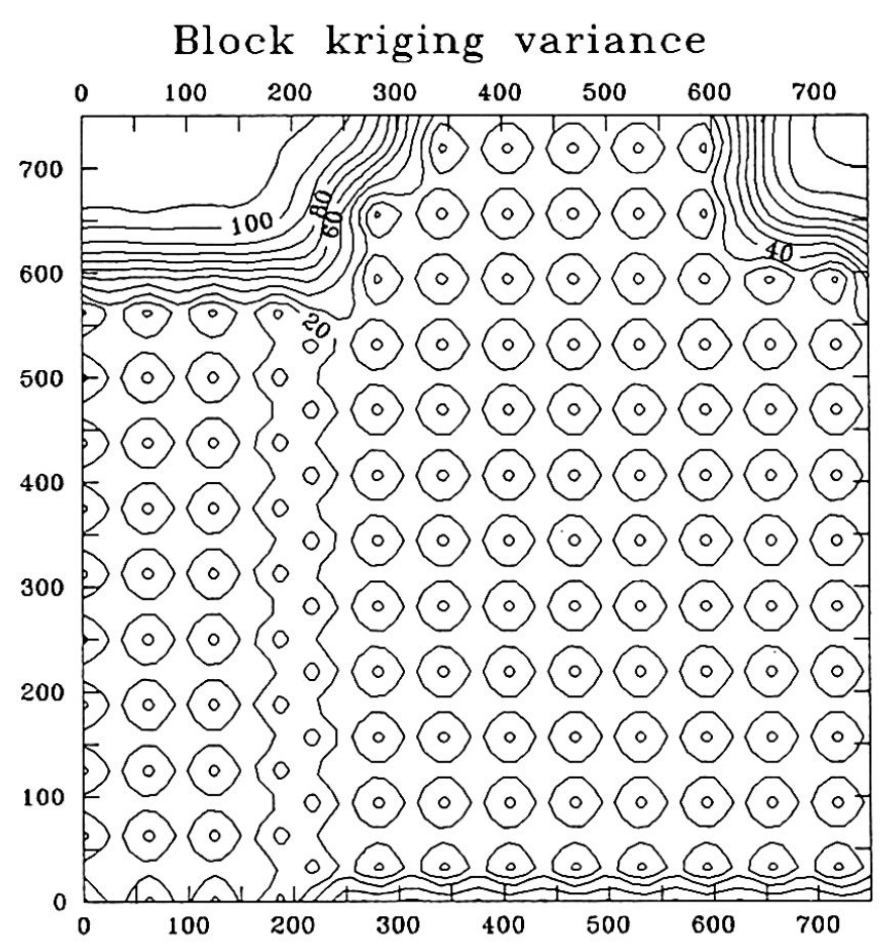

Figur $4 \mathrm{~b}$

4a) Kriging-Interpolation des prozentualen Sandanteils auf einen 30-m-Raster und Isolinieninterpolation mit Hilfe von SplineGlättung. Die originalen Meßstandorte sind durch einen Stern gekennzeichnet. 4b) Fehleroberfläche zu Fig. 4a. Die Schätzfehler sind klein in der Nähe der Datenpunkte $\left(<10 \%^{2}\right)$, wachsen aber in Gebieten ohne Datenwerte bis über $100 \%{ }^{2}$ an. 

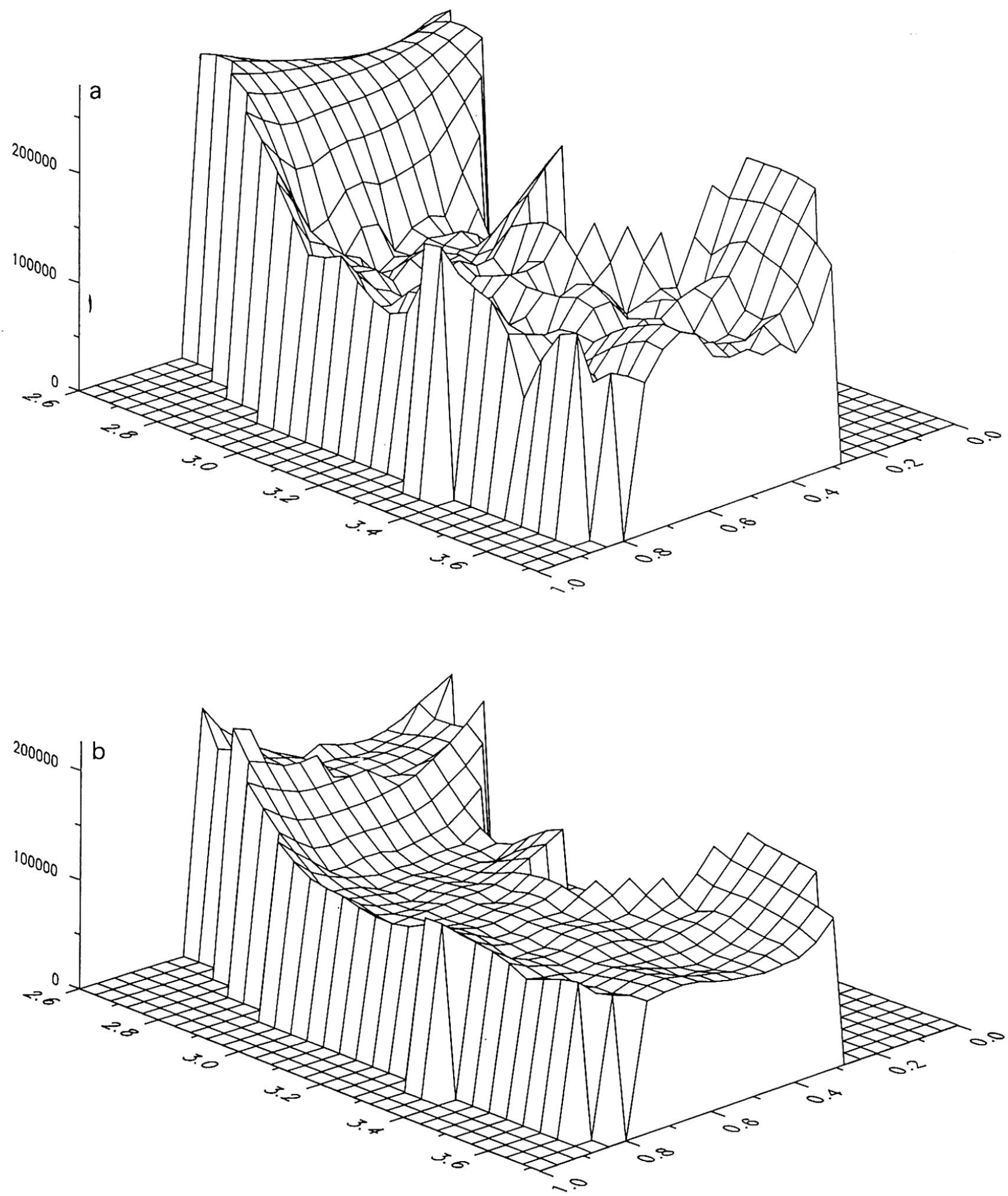

Figur 5

5a) Fehleroberfläche der Interpolation des Zinkanteils in der Flußebene, berechnet durch einfaches Kriging. Im Bereich geringer Stichprobendichten übersteigen die Fehler $300000 \mathrm{mg} / \mathrm{kg}^{2}$. 5b) Fehleroberfläche des Zinkanteils in der Flußebene, berechnet mit Co-Kriging mit zusätzlichen Höhendaten. Der durchschnittliche Fehler wird um $50000 \mathrm{mg} / \mathrm{kg}^{2} \mathrm{reduziert,} \mathrm{der}$ maximale Fehler beträgt noch rund $200000 \mathrm{mg} / \mathrm{kg}^{2}$. 
a) Angaben, ob die Interpolation überhaupt sinnvoll ist (Größe der Nuggetvarianz),

b) Richtungsabhängigkeit der Variation,

c) Größe des Bereiches, innerhalb dessen die Datenpunkte für die Interpolation beigezogen werden sollen und

d) Information zur Bestimmung der Gewichte für eine optimale Schätzung der Attributwerte.

Die wichtigsten Vorteile des Kriging für den Einsatz mit Geographischen Informationssystemen liegen einerseits bei der Möglichkeit, die Interpolation zu optimieren, und andererseits bei der Tatsache, daß man Wahrscheinlichkeits- und Oberflächenfehlerkarten generieren kann. Diese Fehleroberflächen sind besonders dann sinnvoll, wenn interpolierte Daten für die räumliche Modellierung in GIS verwendet werden. Sie sind unerläßlich für die Überwachung der Fehlerfortpflanzung im Modellierprozeß.

Die meisten Kriging-Techniken sind bisher wegen ihrer theoretischen Komplexität, namhafter Rechenansprüche und des Fehlens preisgünstiger Programme nicht als Standardsoftware in GIS integriert worden. Dies ändert sich zurzeit, da jetzt gute und preisgünstige Kriging-Software angeboten wird (ENGLUND und SPARKS 1988).

\section{Verdankungen}

Der Autor bedankt sich bei Dr. H. Leenaers und R.A. Mac Millan für die Beispiele und Prof. K. Brassel für die Übersetzung des Artikels ins Deutsche.

\section{Literatur}

AKIN, H. and SIEMES, H. (1988): Praktische Geostatistik: eine Einführung für den Bergbau- und die Geowissenschaften. Springer-Verlag, Berlin. 304 pp.

BURROUGH, P.A. (1986): Principles of Geographical Information Systems for Land Resources Assessment, Clarendon Press, Oxford.
BURROUGH, P.A. (1989): Matching spatial databases and quantitative models in land resource assessment. Soil Use and Management 5, 3-8.

DAVIS, J. (1986): Statistics and Data Analysis in Geology. 2nd edition, Wiley.

DUMANSKI, J. and ONOFREI, C. (1989): Crop yield models for agricultural land evaluation. Soil Use and Management 5 , 9-15.

ENGLUND, E. and SPARKS, A. (1988): GEO-EAS User's Guide. Environmental Monitoring Systems Laboratory, Office of Research and Development, Environmental Protection Agency, Las Vegas, Nevada.

HEUVELINK, G. B. M., BURROUGH, P. A. and STEIN, A. (1989): Propagation of error in spatial modelling with GIS. Int. J. Geographical Information Systems, 3: 303-322.

JOURNEL, A. G. and HUIJBREGTS, C.J. (1978): Mining Geostatistics. Academic Press, London.

LEENAERS, H., OKX, J.P. and BURROUGH, P.A. (1989): Cokriging: an accurate and inexpensive means of mapping floodplain soil pollution by using elevation data. In: Geostatistics, edited by M. Armstrong. Proceedings of the third Geostatistics Congress, Avignon, October 1988, Kluwer, 371-382.

MACMILLAN, R.A. (1987): A soil survey of the Lacombe Experimental Farm, Alberta. Report Alberta Research Council, Edmonton, Canada.

MATHERON, G. (1971): The theory of regionalised variables and its applications. Les Cahiers du centre de morphologie mathématique de Fontainebleau. Ecole Nationale Supérieure des Mines de Paris.

MCBRATNEY, A. B. and WEBSTER, R. (1983): Optimal interpolation and isarithmic mapping of soil properties: $V$. Co-regionalization and multiple sampling strategy. J. Soil Science, 34: 137-162.

MCBRATNEY, A.B., WEBSTER, R. and BURGESS, T.M. (1981): The design of optimal sampling schemes for local estimation and mapping of regionalized variables: 1 . Theory and method. Computers \& Geosciences 7: 331-334.

WEBSTER, R. and OLIVER, M. (1989): Disjunctive kriging in agriculture. In: Geostatistics, edited by M. Armstrong, Proceedings of the third Geostatistics Congress, Avignon, October 1988, Kluwer, 421-432.

WEBSTER, R. and OLIVER, M. (1990): Statistical Methods in Soil and Land Resources Survey, Oxford University Press. 